\title{
Cartesio: A Software Tool for Pre-implant Stent Analyses
}

\author{
Ciro Indolfi, Mario Cannataro, Pierangelo Veltri, and Giuseppe Tradigo \\ University Magna Græcia of Catanzaro, Germaneto 88100, Italy \\ surname@unicz.it \\ http://www.unicz.it
}

\begin{abstract}
Quantitative angiography has becoming largely used in interventional cardiology thanks to positive outcomes in terms of patients survival indexes and life quality. This technique is based on positioning a medical stent in a laparoscopic intervention to cure coronary stenoses. Patients gain an immediate benefit from such a procedure by avoiding an open-heart surgery procedure. Stent positioning is guided by using contrast liquid and angiographic X-Rays images, which are used to define stent dimensions. Physicians may have difficulties in optimally estimating the stenosis size in order to choose the most appropriate stent mainly because there is no absolute reference on the angiographer screens. In this paper we present an innovative software tool able to assist the physician pre-implant analysis and thus supporting an optimal stent choice.
\end{abstract}

\section{Introduction}

To date, angiography has been the primary tool to asses procedural outcomes after a number of cardiac surgery such as: coronary angioplastic or stent implantation. Its principal role has been to identify major complications (dissections, thrombus) resulting from the procedure. The agioplastic cardiology has changed a lot in the last 40 years. It now makes use of highly advanced clinical and surgery techniques (i.e. laparoscopy, angiographic imaging, biomedical stents). One of the most critical factors of this type of surgery is that patient is awake and conscious of what is going on around him during each step of the clinical activity, during both the disease discovery and the surgery phase. This implies that the patient will immediately feel improvements or aggravations of his health condition and this suggests that decisions should be taken faster than what happened in the past. Nevertheless, in order to avoid stent thrombosis, drug eluting stents, as well as bare metal ones, should be perfectly deployed. Thus the estimation of diameter and length of the coronary vessels as well as overlap of stents at the origin of large collateral branches, is critical. Currently, optimization techniques to physicians in order to measure coronary stenoses are included in software tools coming with angiography equipment. Nevertheless such software tools are either too expensive or difficult in use since the estimation is done via a dedicated catheter to be used just for this application.

G. Allen et al. (Eds.): ICCS 2009, Part I, LNCS 5544, pp. 810-818, 2009.

(C) Springer-Verlag Berlin Heidelberg 2009 
In this paper we present Cartesio, an innovative software tool to be used by physicians working in emodynamics surgery rooms. It helps in making a pre-implant analysis for the estimation of the dimensions of the stent to be implanted. The tool interacts with virtually any angiographic equipment by acquiring its high-resolution video signal and offering a set of functions to zoom, pan, playback, measure and draw a virtual stent over the acquired video frames. Each rendered measurement or stent pre-implant analysis can be exported as a bitmap image on the file system or saved in an experiment repository on a relational database for future reference. The software uses a new patented balloon catheter with radio-opaque iridium markers positioned at $10 \mathrm{~mm}$ from each other.

Cartesio allows the operator to calibrate the images by making the operator click over two radio-opaque markers. After the angiographic images are calibrated the operator can use a software measurement tool to measure distances in millimeters instead of pixels. Furthermore, once the calibration is performed, all of the parameters of the virtual stent (i.e. diameter, length) can be expressed in millimeters. Measurements help physicians to evaluate the exact dimension of the stenosis. After determining such a dimension the physician can set the parameters of the virtual stent and visualize it over the vessel structure. The virtual stent is rendered in real time and can be moved along a previously drawn curve path following the desired vessel. The physician have a precise preview of what will be implanted and, if desired, he can change some parameters or move the stent to better fit the disease. By using such a procedure he can take better and more conscious decisions before the real angioplastic surgery.

A clinical advantage of the system is the visual analysis of radio-opaque markers to establish the stent length and the virtual reconstruction of coronary stent in the angiographic image allowing a further visual double-check analysis before the device is irreversibly implanted.

In this paper we describe the software main features, its architecture and its use in an emodynamics surgery room. Physicians can control the Cartesio tool via a wireless trackball mouse installed at the patient table and visualize its output in one of the monitors connected to the angiographer.

\section{Related Works}

During the last ten years many researchers have exploited novel techniques to extract informations from image data acquired via angiographic equipment. These techniques allow to build models used by physicians in order to take better decisions or to execute calculations (i.e. distance evaluations, angles, vessels diameter) and simulations (i.e. blood flow changes, blood pressure, impact of a vessel dissection).

One of these techniques is Virtual Reality which is used to build geometrical models of real objects in order to perform complex simulations. Once a virtual object has been placed in a virtual scene, often called virtual world, a scientist can define physical parameters both for the object (i.e. mass, color, material) and 
for the world (i.e. gravity, humidity, electric field). Performing simulations in the virtual world is usefull because results are easily reproducible and no real object is involved. Furthermore simulation results can be used to confirm or reject hypotheses. In [14] a complete study about the impact of Virtual Reality simulations over clinical practice is described. Fourty-five experienced interventionalists were recruited and asked to use a commercially available VR simulator to perform a carotid artery stent (CAS) procedure. All subjects rated the simulator highly in terms of realism and training potential and the investigator was able to correlate total and fluoroscopic time of the procedures to the physician's experience level.

Similar investigations about the value of a computer-aided clinical procedures has been explored in various areas of the clinical activity. As an example, we report how similar approaches have been exploited in the neurosurgery field, where biomedical stents are used to cure aneurysms. In [10] a technique for pretreatment planning and visualization of a virtual stent across the aneurysm neck is presented. The authors state that suh a method provides information not otherwise available regarding the location of portions of the stent not visible on fluoroscopy. Furthermore, using the method during a treatment, the operator shows an enhanced ability to determine the location of coils in relation to the stent boundaries, thus avoiding parent artery compromise. In [13] the authors simulate the fluid dynamics effects of positioning multiple stents in the case of an aneurysm in order to prevent a rupture risk. The geometry of a wide-necked saccular basilar trunk aneurysm was reconstructed from a patient's computed tomographic angiography images and three models of commercially available stent were used during simulations. The study showed that The complex flow pattern observed in the unstented aneurysm was suppressed by stenting and the effect was increased by deploying multiple stents.

In literature we can find many papers describing techniques to enhance stent visualization, both in place or virtually, because this has proven to be useful to the physician in deciding how to treat a disease or to evaluate the state or the evolution of a treatment. In [8] the visualization of different coronary artery stent is compared with respect to the detectability of in-stent stenoses during computed tomography in a plastic vessel phantom. The study shows how 16-slice computed tomography is necessary for this task if compared to 4-slice performances. In 9] the ability to assess the coronary artery lumen is measured in the presence of coronary artery stents in multislice spiral computed tomography. In [1] the authors compare some postprocessing techniques for three-dimensional computed tomography in order to visualize normal arterial branches, measure aneurysm diameters and neck lengths as well assessment of vessel patency and presence of endoleaks. In [7] the accuracy of 16-row multidetector computed tomography is measured when used to visualize peripheral artery stents and to the appraise instent stenoses. In [12] a computer program is exploited to plan stent-grafting for thoracic aortic aneurysm with complicated morphology. The program, called Semi Automatic Virtual Stent (SAVS) designer, uses three-dimensional computed tomography data to allow the design of a virtual stent which can be used as a guide to shape a real straight stent to be implanted. 
The IVUS technique [6] is becoming largely used in the emodynamics surgery and allows an estimation of the vessel dimension and shape by using a dedicated catheter that has to be inserted before angioplastic stent positioning. This technique, even if is currently considered the gold optimization technique, has two main disadvantages: it is expensive and requires a pre-analysis phase that has to be performed before inserting the guide with the guide catheter. The use of Cartesio and the radio-opaque marked catheter has the advantage of performing both measurements and guide inserting in the same phase.

Most of the times the angiographer can generate planar images, which means that the physician has to choose the most reliable planar projection of the vessel in order to perform reliable measurements. In order to solve this problem, many efforts have been made by the research community to extract three dimensional models from a pair of planar images taken via the angiographer at different angles. This has lead to two main results: $(i)$ the extraction of a tree of vessels representing connections among vessels and their position in space $[32$; $(i i)$ the creation of a full 3D model with polygons describing vessels narrowings and enlargements, useful to execute punctual simulations of blood flow or just to better visualize stenoses from inside the vessel 114 .

Currently Cartesio has been implemented on planar images and the problem of coronary vessels that are not exactly projected on the image plane can be measured by using local calibration that is always guided by radio-opaque catheter that follows the vessel.

Finally many software tools have been defining by major vendors. For instance the edge-detection support tool from the General Electric workstation allows to measure the diameter and the variation of stenosis profile in the vessels.

\section{The Cartesio Tool}

\subsection{Requirements and Functionalities}

The main phases followed by a phisician during the cardiac surgery activity are:

patient disease discovery phase during which he inserts a catheter through the femoral artery to the heart and, by the injection of a contrast medium to distinguish the vessels shapes, he acquires a set of video sequences with different projections;

disease evaluation phase where the phisician analyzes all the video sequences and frames in order to elaborate and possibly verify a clinical hypothesis on the disease;

plan of action phase in which the medical equipe decides what to do and how, evaluating potential problems and consequences and minimizing the decision time;

intervention phase where the surgery activity is eventually implemented.

The tool has been designed following such phases and offers useful functionalities over the flat angiographic image acquisitions, such as: video signal acquisition, video live, storing, zooming with bi-cubic interpolation, calibration, 


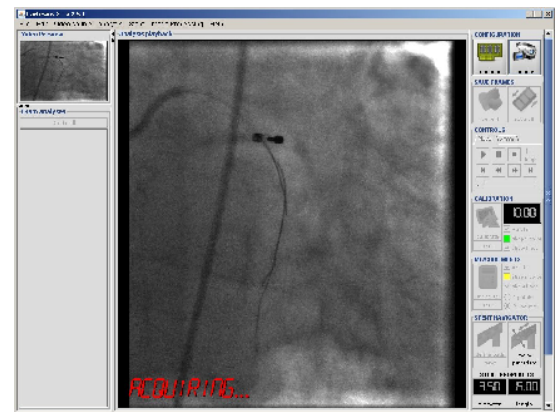

(a) Acquiring video

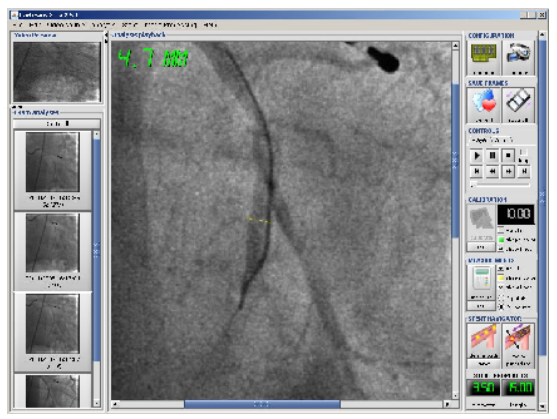

(c) Measuring

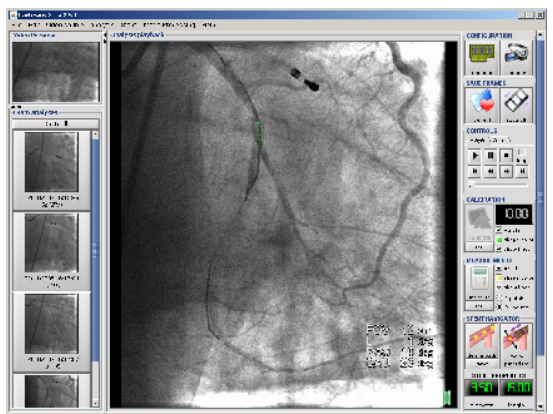

(b) Calibrated

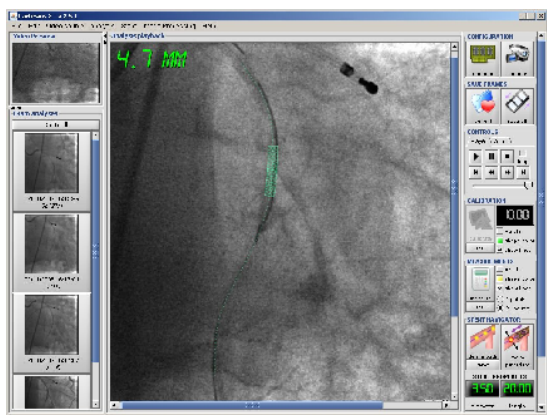

(d) Stent drawing

Fig. 1. Four interaction phases with the Cartesio tool

measurements of the coronary diameter and length. In addition the software is also able to reconstruct in the coronary angiographic segment the stent that operator have selected for a specific lesion. Moreover, it is required to store the data acquired during analysis in the Dicom standard image format on a local repository, data that can be reused for case studies. For instance, in figure 1 . screenshots related to the Acquisition, Calibration, Measuring and Stent drawing and positioning simulation phases are depicted.

The software tool has also been developed to perform procedures both on-line and off-line. In the first case the software tool allows to calibrate and measure vessels and coronaries interactively during the procedure. The second case allows to perform measurements on the images stored in a standard DICOM 11 repository. Many Interventional Units have a shared Picture Archiving and Communication System $(P A C S)$ able to store and retrieve medical images from a large number of equipment in the DICOM format. With the ability to use images stored in the DICOM format, Cartesio offers physicians the ability to perform simulations even after the coronarography analysis, simulating stent positioning and deciding strategies for future treatments.

${ }^{1}$ Digital Imaging and Communications in Medicine (DICOM) is a standard for handling, storing, printing, and transmitting information in medical imaging. It includes a file format definition and a network communications protocol. 
Moreover the Cartesio tool includes classical image manipulation techniques such as: acquiring a video sequence from the angiographer, choosing the best contrast frame on which build the virtual stent model, calibrating the image, measuring distances (i.e. vessel diameters, stenosis diameters), designing the vessel path, choosing the stent parameters and interactively moving it along the designed path.

\section{$3.2 \quad$ Architecture}

The system architecture is structured as a set of components all connected together through a communication bus, as depicted in figure 2 .

Each component of the system implements a common interface enabling it to send and receive messages from the bus. In the initialization phase of the module, it searches for the bus object and makes a subscription. Every other module connected to the bus receives notifications in a publisher/subscriber fashion and our API provides both the pull and the push models. The main Cartesio modules are:

Communication Bus is used by all the other modules to exchange messages and notify events in a publisher/subscriber fashion; when a module initializes itself it registers with the Communication Bus in order to receive and send messages to the other modules;

Command Interpreter together with the User Interface module it is in charge of transforming commands issued by the operator into instructions to be send the other Cartesio modules;

Rendering and Business Logic module implements the logic of the system and offers math, geometric and measuring functionalities to other modules (i.e. conversion functions to transform pixel into millimeters and back); furthermore it holds the state of the system, the data structures and periodically generates rendered images to be shown on the screen by combining bitmap angiographic images with vector data (i.e. curve paths, lines, points, measurement labels);

Video Acquisition module works with both JMF (Java Media Framework) video sources (i.e. low resolution USB video signal) and distributed digital video streams (i.e. native code video components sending acquired video over a TCP/IP network);

Persistence module offers functionalities to import exams in the DICOM format, both for didactica and post-therapy planning, and to export data both on file system, in the XML format, and on a relational database $(R-D B M S)$;

Cartesio is part of a Distributed Electronic Patient Record, in which the patient's personal data and family history are enriched with clinical files and DICOM images documenting exams results. The patient files are included during the coronarography planning and all files with biological data and DICOM images are included in the Electronic Patient Record for future reference.

Main modules are depicted in figure 2. In the following we briefly discuss the Video Acquisition module and we sketch the Rendering module as the main 


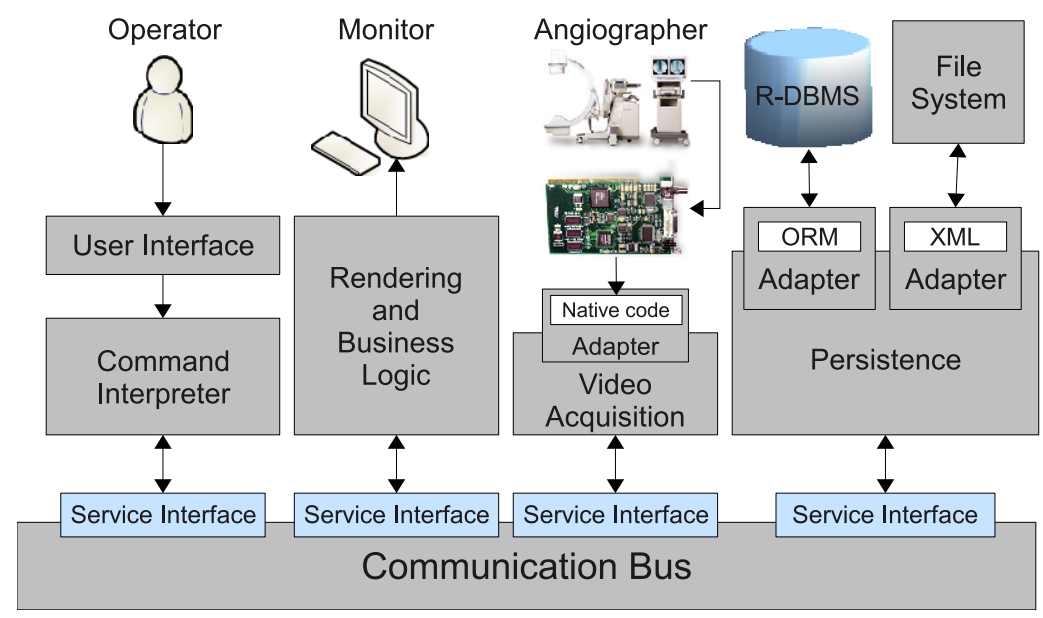

Fig. 2. Module architecture of the Cartesio tool

algorithmically challenging. The acquisition module consists in acquiring an high resolution and high frequency analog video signal generated from angiographic equipment. The lack for a video signal standard in medical applications have lead to a plethora of different specifications, which makes really hard to design an acquisition platform working for every equipment, even for instruments from the same vendor. We managed to acquire all of the video signals generated by equipment found in our structure, by using the following strategy:

1. measuring peak-peak voltage, identifying the video sync signal and resolution;

2. identifying the acquisition signal frequency;

3. tuning the acquisition module in order to correctly hook the video signal and in order to acquiring data in main memory.

The data volume generated during the video signal digitalization is, in generale, very large. For instance, for the General Electrics Innova System, the acquired full-resolution video signal generates a data flow of approximately 20 Megabytes per second. For this reason, while Cartesio has been implemented using the Java language, the video acquisition component has been coded in native code in order to maximize the frame rate. After the signal has been correctly grabbed and digitalized, it is made available to Cartesio. Note that, due to the large amount of data, an ad-hoc communication channel between the image acquisition native component and the Cartesio image manipulation module has been designed.

The Rendering module is in charge to position and visualize vector data over the bitmap angiographic images in Cartesio. It interpolates the stent path, designed by the physician across a vessel of interest, through a set of Bezier curves 5] connecting a set of control points. By moving or adding control points on the angiographic image, the interpolation curve is recalculated in real time. Along 
the curve path the operator will be able to move and resize the virtual stent. The stent shape will be visible after the definition of the stent diameter and length, by calculating two bezier curves equidistant from the main stent path.

\section{Experiments and Conclusions}

Since June 2008 the Cartesio tool has been testing in Emodynamic Department of University "Magna Græcia" of Catanzaro Medical School Hospital. As a first setup Cartesio has been installed on a $2 \mathrm{~Gb}$ RAM quad core computer with Microsoft Window operating system. The video signal has been acquired from the video output of an Rx machine General Electrics Innova 2100. The system has been installed in the emodynamics surgery room and can be controlled both by the physician during the interventional procedure or by a technician outside the operating room. According to our tests, Cartesio measurement facilities and simulations improve the evaluation of correct stent choice thus optimizing the interventional procedure in terms of time and confidence. The improved capability in correctly evaluating the stent dimensions has been confirmed by expert operators. The only drawback is mainly that the physician has to choose a correct projection for acquiring the angiographic images (i.e. planar w.r.t. the diseased coronary) in order to allow uniform measurement analyses through the whole image. The software has also been tested with the italian SIAS angiographer and also on the General Electrics Advantic, with similar performances.

We conclude claiming that Cartesio is a software tool that both enhances the real-time visualization of the angiographic images and offers new image processing functionalities supporting the surgery procedures. Currently the tool is in use in two surgery rooms in the University of Catanzaro Medical School Hospital, and data have been storing in the Cartesio DICOM database. We are improving the software tool by adding multiple point calibration and designing a collaborative version allowing the operator to share the virtual stent simulation environment with remote collegues. Such a collaborative environment could bring new emodynamic techniques to remote hospital or clinical structures not having the expertise in using novel or advanced angioplastic techniques.

\section{References}

1. Yang, J., Wang, Y.T., Liu, Y., Tang, S.Y., Li, Y.H.: 3-D Reconstruction of Coronary Arterial Trees from Uncalibrated Monoplane Angiographic Images. Chinese Journal of Computers 30, 1594-1602 (2007)

2. Kerrien, E.: Outils d'imagerie multimodalité pour la neuroradiologie interventionnelle. Phd Thesis, Institut National Polytechnique de Lorraine (2007)

3. Kerrien, E., Vaillant, R., Launay, L., Berger, M.O., Maurincomme, E., Picard, L.: Machine precision assessment for $3 \mathrm{D} / 2 \mathrm{D}$ digital subtracted angiography images registration. In: Hanson, K. (ed.) Proceedings of SPIE Medical Imaging, vol. 3338, pp. 39-49 (1998) 
4. Ogiela, M.R., Tadeusiewicz, R., Trzupek, M.: Graph-Based Semantic Description in Medical Knowledge Representation and 3D Coronary Vessels Recognition. In: Indulska, J., Ma, J., Yang, L.T., Ungerer, T., Cao, J. (eds.) UIC 2007. LNCS, vol. 4611, pp. 1079-1088. Springer, Heidelberg (2007)

5. Knuth, D.: Metafont: the Program, pp. 123-131. Addison-Wesley, Reading (1986)

6. Safian, R.D., Freed, M.S.: Intravascular Ultrasound. The Manual Of Interventional Cardiology, 712. Physicians Press (2002)

7. Herzog, C., Grebe, C., Mahnken, A., Balzer, J.O., Mack, M.G., Zangos, S., Ackermann, H., Schaller, S., Seifert, T., Ohnesorge, B., Vogl, T.J.: Peripheral artery stent visualization and in-stent stenosis analysis in 16-row computed tomography: an in-vitro evaluation. European Radiology 15, 2276-2283 (2005)

8. Maintz, D., Seifarth, H., Flohr, T., Kramer, S., Wichter, T., Heindel, W., Fischbach, R.: Improved Coronary Artery Stent Visualization and In-Stent Stenosis Detection Using 16-Slice Computed-Tomography and Dedicated Image Reconstruction Technique. Investigative Radiology 38, 790-795 (2003)

9. Mahnken, A.H., Buecker, A., Wildberger, J.E., Ruebben, A., Stanzel, S., Vogt, F., Gunther, R.W., Blindt, R.: Coronary Artery Stents in Multislice Computed Tomography: In Vitro Artifact Evaluation. Investigative Radiology 39, 27-33 (2004)

10. Karmonik, C., Strother, C.M., Chen, X., Deinzer, F., Klucznik, R., Mawad, M.E.: Stent-Assisted Coiling of Intracranial Aneurysms Aided by Virtual Parent Artery Reconstruction. American Journal of Neuroradiology 26, 2368-2370 (2005)

11. Sun, Z., Winder, R.J., Kelly, B.E., Ellis, P.K., Kennedy, P.T., Hirst, D.G.: Diagnostic Value of CT Virtual Intravascular Endoscopy in Aortic Stent-Grafting. Journal of Endovascular Therapy 11, 13-25

12. Hyodoh, H., Katagiri, Y., Sakai, T., Hyodoh, K., Akiba, H., Hareyama, M.: Creation of individual ideally shaped stents using multi-slice CT: in vitro results from the semi-automatic virtual stent (SAVS) designer. European Radiology 15, 1623$1628(2005)$

13. Kim, M., Levy, E.I., Meng, H., Hopkins, L.N.: Quantification of Hemodynamic Changes Induced By Virtual Placement of Multiple Stents Across A Wide-Necked Basilar Trunk Aneurysm. Neurosurgery 61, 1305-1313 (2007)

14. Van Herzeele, I., Aggarwal, R., Choong, A., Brightwell, R., Vermassen, F., Cheshire, N.: Virtual reality simulation objectively differentiates level of carotid stent experience in experienced interventionalists. Journal of Vascular Surgery 46, 855-863 (2007) 